\title{
INSTRUMENTATION \& TECHNIQUES A head-mounted scene holder for eye movement research
}

\author{
CRIS W. JOHNSTON and J. THOMAS HUTTON \\ Neurology Service and Geriatric Research, Education, and Clinical Center and \\ Department of Neurology, University of Minnesota, Minneapolis, Minnesota 55417
}

\begin{abstract}
An inexpensive head-mounted scene holder is described. In contrast to the usual biteboard/fixed-head approach, the present apparatus allows head movements without introducing substantial artifact. This enables eye movement investigations of brain-damaged or psychiatric subjects who often cannot maintain prolonged head immobility; also, the unit makes possible subjects' verbal reports coincident with visual scanning.
\end{abstract}

Head immobilization techniques generally used in eye movement research are inadequate or inappropriate for some types of studies. Experiments with certain patient populations are difficult to perform using the standard bitebar or chin-cup/head-rest apparatus. Subjects with impaired cognitive abilities, shortened attention span, poor memory, or discomfort associated with illness seldom sit still for long periods. In addition to subject variables, paradigms requiring verbal responses simultaneously with eye movement recordings are problematic, as the jaw or chin serves as a point of restraint. Consequently, verbalizations bring about head movement and thereby invalidate the calibration. This paper describes a headmounted visual target holder designed to overcome these limitations of standard immobilization techniques.

\section{APPARATUS DESCRIPTION ${ }^{1}$}

The head-mounted visual target holder consists of a counterbalanced scene holder assembly attached to a standard welder's headgear. The welder's headgear ${ }^{2}$ has an adjustment for head circumference and a coronal adjustment strap and two metal plates suitable for attaching the scene holder assembly (see Figure 1).

A small stainless steel bracket is attached to each metal plate of the headgear. An aluminum collar is held by screws to each of the brackets. The scene holder assembly slides through the collar attaching it to the headgear. This allows adjustment of the distance from the card holder to the subject's eyes. Set screws tighten the collar to prevent the scene holder assembly from sliding once the desired position is found.

The scene holder assembly consists of a card holder,

This research was supported by the Veterans Administration. Reprint requests should be mailed to the authors, Department of Neurology, VA Hospital, 54th Street and 48th Avenue South, Minneapolis, Minnesota 55417. two long aluminum support tubes, and a counterweight. One portion of the card holder is fashioned from a sheet of light-gauge aluminum on which nine light-emitting diodes (LEDs) are mounted (see Figure 2). The LEDs are in a 3-row by 3-column grid and are used for calibration. The aluminum sheet is secured to a U-shaped frame made of light-gauge, hollow aluminum tubing. Three stainless steel strips are also attached to the U-shaped frame. The strips are molded to provide a grooved guide to support visual targets which are mounted on light tagboard.

The upper right and left corners of the card holder (the ends of the U-shaped aluminum frame) are welded to one end of two $86-\mathrm{cm}$-long small-diameter hollow aluminum tubes. A small brace made of light-gauge aluminum is screwed to each member at these junctures to provide additional strength. Wires connecting the LEDs to a power supply and control box are routed through the tubes and exit to a connector at the rear.

The rear ends of the tubes are joined by a square aluminum rod (see Figure 1). Extending back from the center of the square rod is a stainless steel stem $29 \mathrm{~cm}$ long. A $200 \mathrm{~g}$ sliding counterweight is mounted on the stem to offset the weight of the card holder.

In order to shield the subject's eyes while changing stimulus scenes, a drop cloth is suspended from the scene holder assembly directly in front of the subject's eyes using a stiff fine wire sewn into the top of the drop cloth. When in place, the drop cloth interrupts a light beam from a subminiature lens-end light directed at a photosensor, both of which are mounted on one of the long tube supports (see Figure 2). When the drop cloth is lifted, the photocell receives the light beam, thus creating a pulse which indicates the instant the scene is exposed to the subject. Wiring for this marker pulse is routed through to the rear of the aluminum tube.

The weight of the entire headgear, including wiring and cable connectors, is $680 \mathrm{~g}$. Major pressure points of the head-size adjustor are lined with foam padding. 


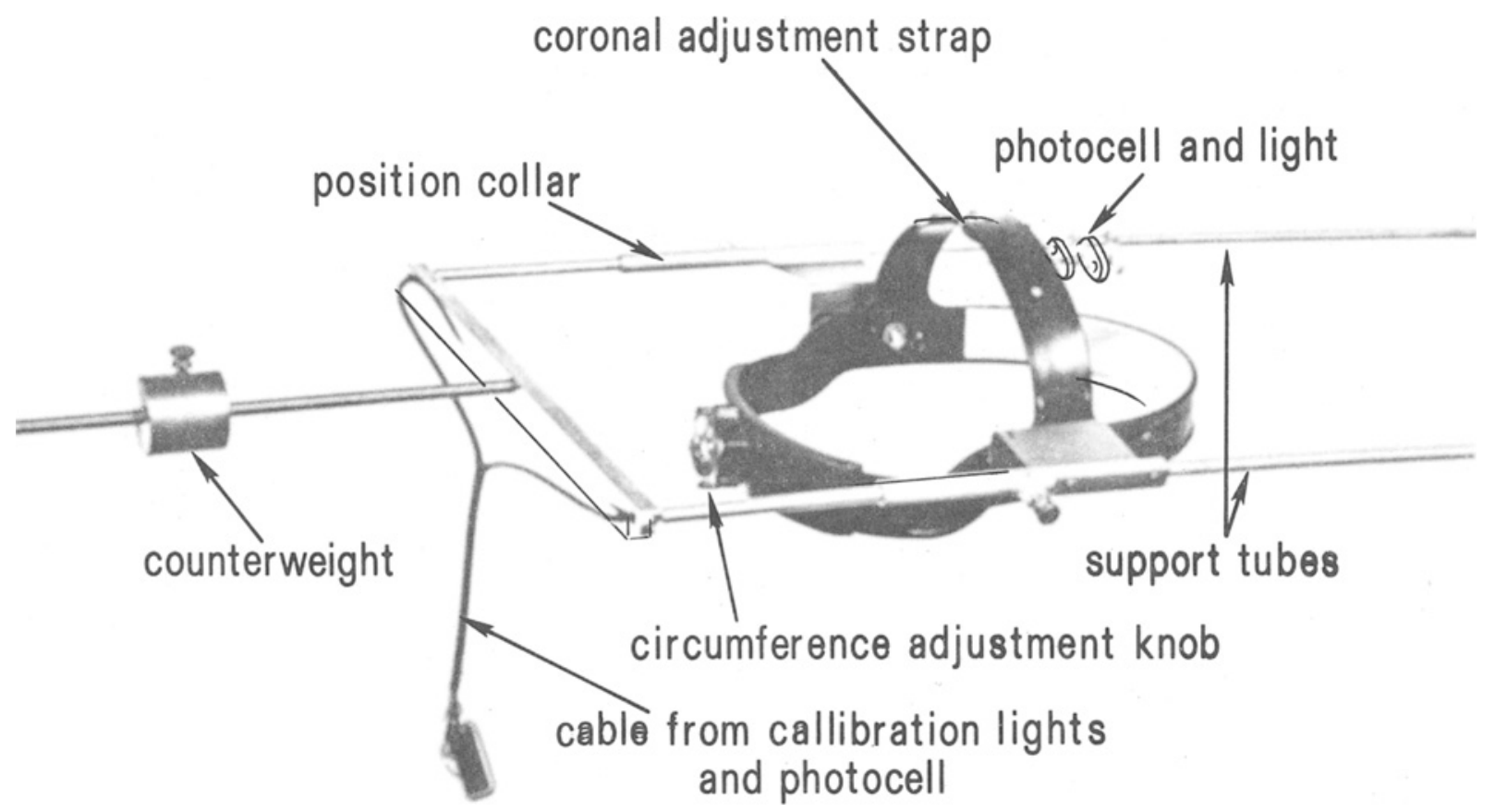

Figure 1. Head-mounted scene holder showing welder's headgear, counterweight, positioning of photocell, and support tubes.

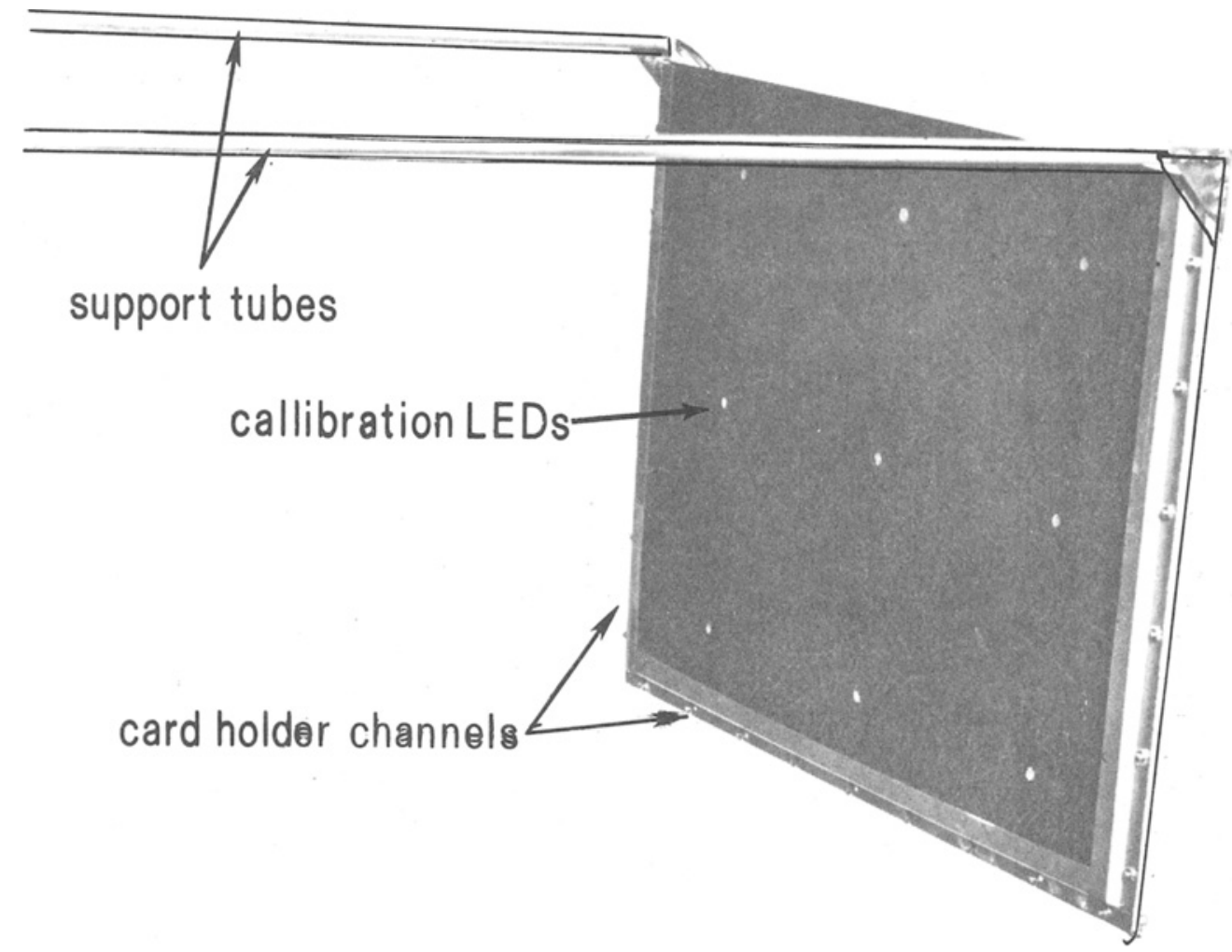

Figure 2. Head-mounted scene holder showing card holder, calibration LEDs, and support tubes. 
Cable and connector weight may be relieved by pinning to the subject's shirt. Approximately $30 \mathrm{~h}$ are required to construct the apparatus.

\section{PERFORMANCE CHARACTERISTICS}

Several tests were performed to assess the stability of the head-mounted scene holder. In each case, eye movement data were obtained using a spectacle mounted infrared photoelectric technique. ${ }^{3}$

Figure 3 demonstrates the effect of talking vs. no talking for two conditions: eye fixation and eye movement. In Figure $3 a$, the subject is staring silently at a central fixation light subtending $20 \mathrm{~min}$ of visual arc. In Figure 3b, the subject is fixating the same target while reciting the days of the week. Note that, in both recordings, the vertical plane shows less accuracy than does the horizontal, probably because the vertical eye movement signal is taken from the lower eyelid. This requirement of the recording apparatus results in an accuracy of 2 deg vertically as compared to $1 \mathrm{deg}$ horizontally. ${ }^{4}$ The basis for this difference is that vertical pseudoeye movements are recorded when the subject squints or widens his eyes; the same but smaller artifacts occur in some subjects (younger individuals with tighter skin) while uttering sounds that demand a pursing of the lips. Considering the drawbacks of the eye monitor, it is evident that the head-mounted scene holder does not produce an eye movement recording that is artifactual beyond the performance specifications of the eye monitoring device itself.

Figure $3 \mathrm{c}$ is an example of tracing a square without talking, and Figure 3d is th same task while the subject recites the days of the week. These figures demonstrate the negligible effect talking has on a scan pattern for a
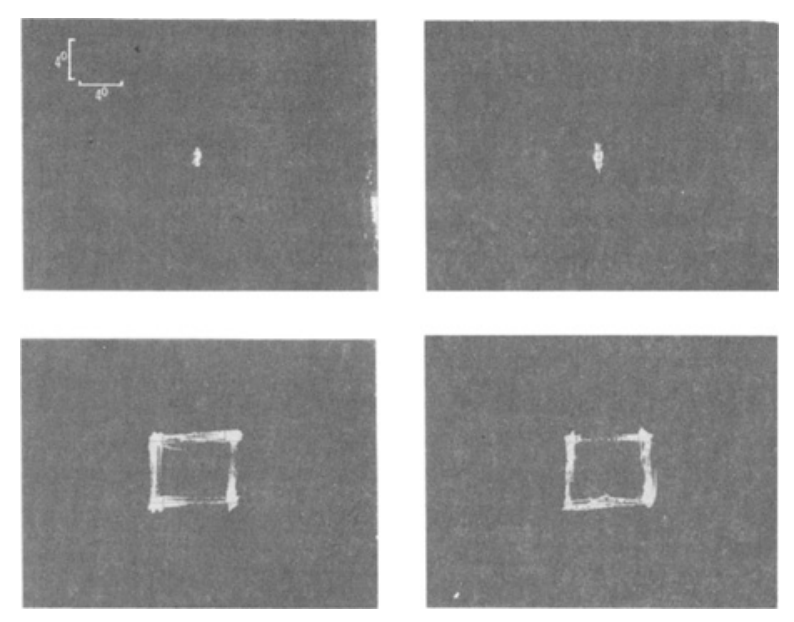

Figure 3. (a) Ten-second fixation of a point target without talking. (b) Ten-second fixation of the same target with talking. (c) Visually trac:ng a squarc for $10 \mathrm{sec}$ without talking. (d) Visually tracing a square for $10 \mathrm{sec}$ with talking.

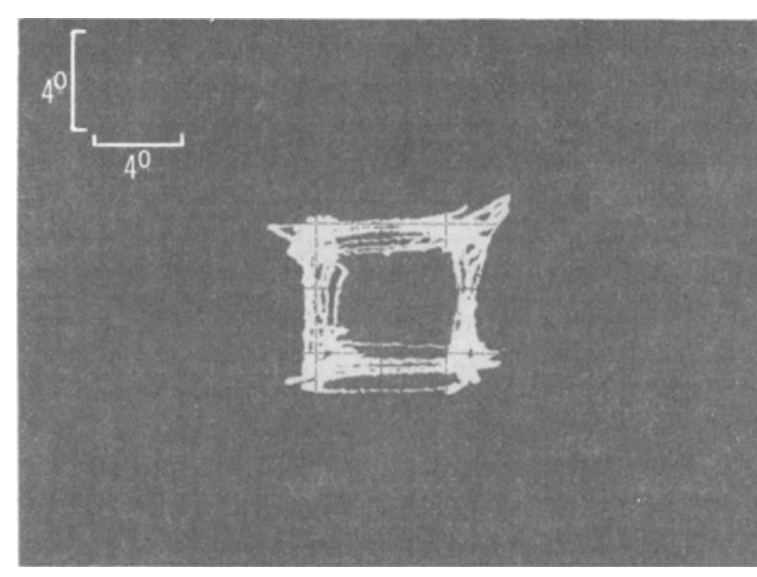

Figure 4. Visually tracing a square for $10 \mathrm{sec}$ and moving the head at the same time and in the same direction of each eye movement.

picture that has been placed in the head-mounted scene holder.

Figure 4 shows the effect of moving the head concurrent with, and in the same direction of, each saccade while tracing a square. The subject is silent during this task. Head movements are more abrupt and cover a greater distance than typically occurs even with the most fidgety and uncooperative subjects. The movements are the speed of an orienting response and cover a distance of approximately $40 \mathrm{deg}$ in each direction. Even with such extreme movement, a proportional outline of the square is well preserved.

To determine the duration of wobble resulting from such an abrupt head movement, and its effect on eye movements, a strip-chart recording was made to display horizontal and vertical eye position and horizontal and vertical scene holder tremor (see Figure 5). Scene holder tremor was recorded by securing an accelerometer $^{5}$ to the distal end of the head-mounted scene holder. An abrupt horizontal movement (similar to that in the tracing task) was made first in one direction, followed by a pause until the apparatus had settled, then in the other direction. The subject attempted to maintain a central fixation at all times. Under these conditions three to fuur major eye movements occur over the duration of the head movement and settling period of the apparatus. The settling period in this case lasts approximately $2 \mathrm{sec}$. Although extreme head movements contaminate eye movement data during the event, at the conclusion of each event the original eye position is retained.

Figure 6 represents the calibration of the eye monitor using the nine calibration lights attached to the scene holder. Tracing Figure 6a shows the calibrated coordirate fixations obtained prior to performing all the above experiments. Whereas $6 \mathrm{a}$ is the actual calibration, $6 b$ is a repeat performance of the same eye movement task obtained at the completion of the above experiments. The repeat checks the stability of the head- 


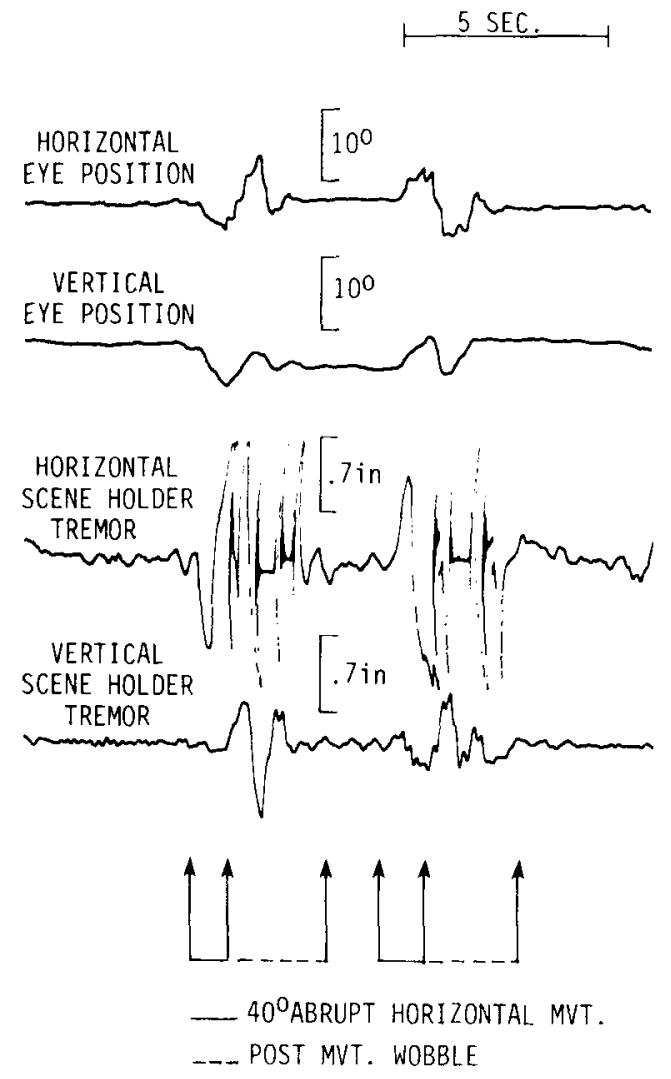

Figure 5. The effect of abrupt head movements while attempting to maintain fixation of a point target. Channels $I$ and 2 denote horizontal and vertical eye position, Channels 3 and 4 reflect amount of horizontal and vertical tremor of the headmounted scene holder. Calibration of the accelerometer to record wobble of the head-mounted scene holder does not permit a linear representation of movement greater than \pm 1.0 in. Thus, the large (40-deg) major movement shown on the stripchart recording is disproportionate to the terminal movements of interest.

mounted scene holder once it is attached. The only plane in which any coordinates changed was the vertical. The change was minimal, and the explanation for the degree of inaccuracy was previously provided.

\section{DISCUSSION}

To date, most eye movement research of psychological processes has utilized the fixed head-holder approach, as this appears to provide the most accurate recordings (Mackworth, 1976). With most cooperative nonhospitalized subjects, a chin cup and head-bracing device appear sufficient. With less cooperative normal subjects, or where greater precision is required, bite boards often are employed.

Only occasional attempts have bein made to apply the technology of eye movement monitoring to patient populations in which cooperation is not assured (Tyler, 1969; Yarbus, 1967). The limited research on neurological, psychiatric, or even normal elderly subjects appears, in part, to be due to limitations of apparatus to relate to the special problems of such subjects, namely, an inability or insufficient motivation to maintain prolonged immobility of the head.

Various techniques use a stationary observation field yet allow some head motion; these are said to introduce only slight inaccuracies in the recording (Young \& Sheena, 1975). One method monitors head position and then corrects for head movement during the data processing phase. Another approach utilizes a recording device designed to automatically compensate for head movements occurring during data acquisition. Possible objections to the two approaches include increased data handling and expense.

Although the apparatus described in this report was designed to be used with a relatively simple and inexpensive infrared system mounted on a pair of spectacle frames, it can also be applied to other recording techniques (e.g., EOG). The subject wearing this device is not required to remain in a fixed head position during eye movement recording, and the system permits unhampered verbalization during data acquisition. These
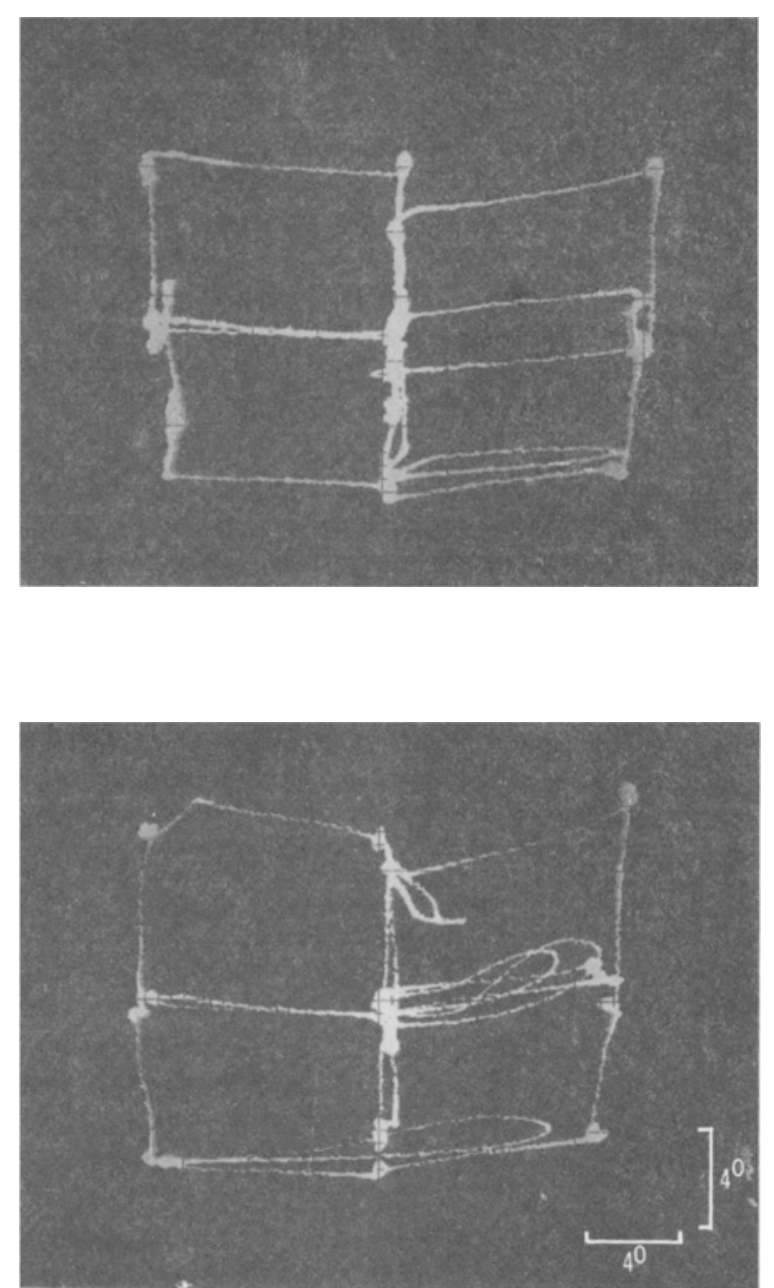

Figure 6. (a) Scan pattern of calibration coordinates prior to testing performance characteristics of the head-mounted scene holder. (b) Scan pattern of calibration coordinates at conclusion of experiment. 
advantages serve to broaden the scope of possibilities for applying eye movement recording to a variety of experimental situations.

\section{REFERENCES}

Mackworth, N. H. Ways of recording line of sight. In R. A. Monty \& J. W. Senders (Eds.), Eye movements and psychological processes. Hillsdale, N.J: Erlbaum, 1976.

TYLER, H. R. Disorders of scanning with frontal lobe lesions. In S. Locke (Ed.), Recent advances in neurology. Boston: Little, Brown, 1969.

YARBUS, A. L. Eye movements and vision. (Translated by Basil Haigh.) New York: Plenum, 1967.

Young, L. R., \& Sheena, D. Survey of eye movement recording methods. Behavior Research Methods \& Instrumentation, 1975, 7, 397-429.

\section{NOTES}

1. The authors would like to thank Vern Nelson for constructing the head-mounted scene holder.

2. Huntsman Headgear No. 117, Kedman Company, P.O. Box 25667, Salt Lake City, Utah 84125 .

3. Eye-Trace Model 200, Gulf+Western Applied Science Laboratories, 335 Bear Hill Road, Waltham, Massachusetts 02154.

4. Performance specifications of Eye-Trace Model 200, Operator's Manual.

5. The authors would like to thank David Webster, M.D., for loan of the accelerometer, a device he designed to accurately document the frequency and amplitude of extremity tremor. This apparatus consists of a lightweight transducer which, in its intended use, is attached to one of the digits of a hand and to an amplifier and electronic circuitry which provides an analog output as shown in Figure 5.

(Received for publication December 28, 1978;

revision accepted May 3, 1979.) 